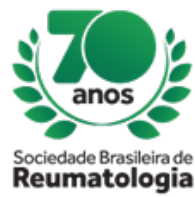

\title{
HOSPITALIZATIONS AND DEATHS BY RHEUMATOID ARTHRITIS AND OTHER INFLAMMATORY ARTHROPATHIES IN BRAZIL 2008-2018
}

João Carlos Geber Júnior (Departamento de Clínica Médica - Hospital Alvorada Brasília, Brasília, DF, Brasil), Andressa Freitas Gomide de Araújo (Faculdade de Medicina - Centro Universitário de Brasília, Brasília, DF, Brasil), Aline Rizzo Borges (Faculdade de Medicina - Centro Universitário de Brasília, Brasília, DF, Brasil), Danyella Oliveira de Paula (Faculdade de Medicina - Centro Universitário de Brasília, Brasília, DF, Brasil), Viviane Cristina Uliana Peterle (Faculdade de Medicina - Escola Superior de Ciências da Saúde, Brasília, DF, Brasil), Ana Paula Monteiro Gomides Reis (Faculdade de Medicina - Centro Universitário de Brasília, Brasília, DF, Brasil)

\section{BACKGROUND}

Rheumatoid arthritis (RA) is a systemic autoimmune inflammatory disease whose prevalence is 0.5 to $1 \%$ in the adult population worldwide, with a peak incidence between the third and fifth decade of life. Due to its chronic character and irreversible joint damage to RA, as well as other inflammatory arthropathies, can generate great functional limitation and impact on the quality of life of the patient, attending with high direct and indirect costs for the individual and public health. The objective of this study was to evaluate hospital admissions as well as mortality from these diseases in Brazil between 2008 and 2018.

\section{MATERIALS AND METHODS}

Descriptive, ecological study, in a time series, based on data obtained from the Hospital Information System of the Unified Health System (SIH-SUS). Data were collected from hospitalizations for RA and other inflammatory arthropathies (ICD-10 M05-M14) of patients over 20 years of age from 2008 to 2018. The following variables were analyzed: number of hospitalizations, total value, value mean of hospital stay, deaths and mortality rate.

\section{RESULTS}

A total of 165,158 admissions were performed, with 1: 1.12 being distribution rate between women and men. The number of hospitalizations and deaths by age group to be seen in table 1 . The average of hospitalization time was 5.1 days and the mean value of $R \$ 780.43$ each, generating a total cost of $R \$$ 128.9 million. This value corresponds to $4.1 \%$ of the hospitalizations for ICD-10 Systemic Diseases of the Heart and Connective Tissue and $0.12 \%$ of all admissions in the period studied. Of the 27 Federative Units (UFs), five of them - São Paulo, Pará, Bahia, Paraná and Goiás - accounted for $37 \%$ of hospitalizations. Ten of the UFs - Pará, Maranhão, Pernambuco, Minas Gerais, Espírito Santo, São Paulo, Paraná, Santa Catarina, Rio Grande do Sul and Goiás accounted for $82 \%$ of total costs. The state of Paraná stood out for having the highest average value for hospitalization $\mathrm{R} \$ 1,439.66$, larger almost double the national average. Roraima had the longest average length of stay of 9.4 days.

\section{CONCLUSION}

Rheumatoid arthritis and other unspecified inflammatory arthritis are responsible for hospitalizations, deaths and high costs in Brazil. New studies like this should be encouraged to better understand the impact of these diseases on the national scene. 